\title{
Interfacial Dynamics in Supported Ultrathin Polymer Films - From the Solid to the Free Interface
}

Emmanuel Urandu Mapesa ${ }^{\dagger}$, Nobahar Shahidi ${ }^{\dagger}$, Friedrich Kremer ${ }^{\ddagger}$, Manolis Doxastakis ${ }^{+*}$ \& Joshua Sangoro $^{+*}$

$\dagger$ Department of Chemical and Biomolecular Engineering, University of Tennessee Knoxville, 1512 Middle Dr., Knoxville, TN-37996, USA.

†Department of Molecular Physics, Peter Debye Institute of Soft Matter Physics, University of Leipzig, Linnéstr. 5, 04103 Leipzig, Germany. 


\section{Experimental and Simulation Details}

1.1 Experimental. Poly(vinyl acetate) (PVAc) samples with molecular weight, $\mathrm{M}_{\mathrm{w}}$, equal to 110 $\mathrm{kg} / \mathrm{mol}$ (PDI = 2, Polymer Source Inc.) and $157 \mathrm{~kg} / \mathrm{mol}$ (PDI = 2.3, Scientific Polymer Products) were used as received without any further purification. Chloroform (purity $\geq 99.8 \%$, Sigma Aldrich) was used as solvent to dissolve the polymers. The procedure used for film preparation is published elsewhere. ${ }^{2}$ Bearing in mind that the boiling point of chloroform is $335 \mathrm{~K}$ and that PVAc degrades in vacuum at temperatures above $500 \mathrm{~K},{ }^{3}$ the films were annealed at $400 \mathrm{~K}$ for $24 \mathrm{~h}$ in an oil-free vacuum $\left(10^{-6} \mathrm{mbar}\right)$ in order to relax them and remove solvent. All films reported in this work had a post-BDS measurement root mean square roughness less than $2 \mathrm{~nm}$ on a scan area of $20 \mu^{2}$. For dielectric investigations, the sample capacitors containing thin films were assembled using a nanostructured electrode arrangement (see schematic in Inset, Fig. 1b) where a regular array of highly insulating silica nano-pillars (base area $5 \mu \mathrm{m}$ x $5 \mu \mathrm{m}$, height $\sim 70 \mathrm{~nm}$, and an inter-pillar separation of $45 \mu \mathrm{m}$ ) serves as separation to ensure electrical isolation. Ultraflat (rms roughness of $0.6 \mathrm{~nm}$ on a micrometric scale) and highly conductive (resistivity $<5 \mathrm{~m} \Omega \mathrm{cm}$ ) silicon wafers are used as electrodes. For detailed information on the use of this capacitor configuration and cleaning procedures, the reader is referred previous publications by some of the current authors $^{2,4-7}$. Bulk samples were measured using $20 \mathrm{~mm}$ stainless steel electrodes in a parallel-plate configuration with $100 \mu \mathrm{m}$ teflon spacers to maintain sample thickness. All dielectric measurements were carried out on a high resolution Novocontrol Alpha Analyzer (frequency range $10^{-1}-10^{7} \mathrm{~Hz}$ ) and the temperature control regulated by a QUATRO system (Novocontrol) using a jet of dry nitrogen, thereby ensuring relative and absolute errors better than 0.1 and $2 \mathrm{~K}$, respectively.

1.2 MD Simulations. Molecular dynamics (MD) simulations were performed with the GROMACS 2018.3 package.$^{8-9}$ Atactic poly(vinyl acetate) was modeled with the atomistic OPLS-AA forcefield for hydrocarbons. ${ }^{10-11}$ An atomistic model of amorphous silica was employed to simulate the oxidized layer in contact with the films in BDS experiments. The nonbond parameters for silica were taken from Berendsen et $a l .{ }^{8}$ and Ndoro et al. ${ }^{12}$; the bonded parameters and initial structure were adopted from Pandey and Doxastakis ${ }^{13}$, and hydrogens added to the structure. The simulations were performed with a 2 fs time step with semi-isotropic pressure coupling using the Berendsen barostat ${ }^{14}$ and stochastic velocity rescaling thermostat ${ }^{15}$ with a time step of 1.0 ps and $0.1 \mathrm{ps}$, respectively. The box depth in the $z$ dimension was kept constant to include vacuum on top of PVAc. Non-bonded interactions were switched to zero at $1.3 \mathrm{~nm}$ and a reaction field was employed for the coulombic interactions with the relative dielectric constant of $\varepsilon_{r f}=3.8$ (taken from the average experimental dielectric constant of silica and poly(vinyl acetate) at room temperature ${ }^{12,16}$ ). The bulk with $\mathrm{N}=10$ monomers has a density of $1073 \mathrm{~kg} / \mathrm{m}^{3}$ at $400 \mathrm{~K}$ which is in agreement with experimental value of $1107 \mathrm{~kg} / \mathrm{m}^{3} .{ }^{17}$ PVAC films each contained 614 chains with 10 repeat units $(\mathrm{Mw}=0.86 \mathrm{~kg} / \mathrm{mol})$ and were simulated at temperatures of 560, 530, 500, 480, 460, 430 and $400 \mathrm{~K}$ at $1 \mathrm{bar}$. The films were each equilibrated for 200, 250, 300, 450, $450 \mathrm{~ns}$ and $1.5 \mu$ s resulting in thin films with thickness ranging from 13.4 to $17 \mathrm{~nm}$, as the temperature decreases. 


\section{Typical dielectric spectra of bulk and ultrathin PVAc films}

The normalized dielectric data for the series of $157 \mathrm{~kg} / \mathrm{mol}$ polymer films is given below (Fig. S1) to highlight the broadening of the distributions of relaxation times with decreasing film thickness. A comparison of the experimental and simulations data is also given in Figure S3.

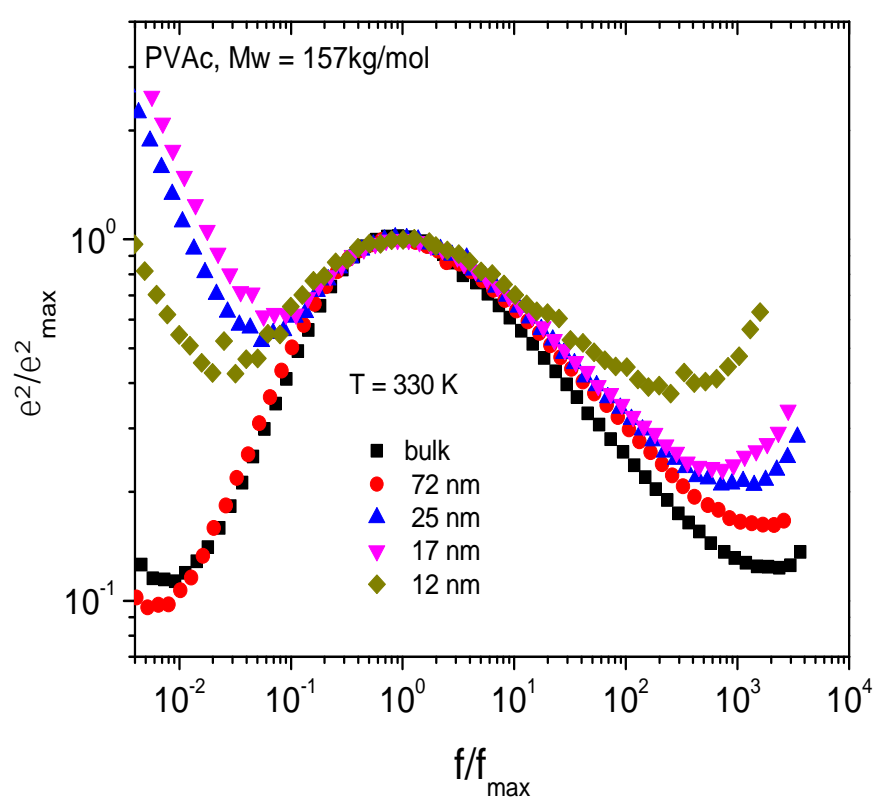

FIGURE S1. Data measured at $330 \mathrm{~K}$ showing the dielectric loss data as a function of frequency normalized with respect to the maximum loss for PVAc $(\mathrm{Mw}=157 \mathrm{~kg} / \mathrm{mol})$ samples ranging in size from bulk dimensions (thickness, $\sim 100 \mu \mathrm{m})$ to thin films, with thicknesses as indicated.

\section{Reorientation of Backbone Torsion}

The backbone dihedral autocorrelation is compared with the monomer dipole autocorrelation in Figure S2. Although the main component of the dipole is located in the sidechain, the re-orientation of the backbone plays an important part in the segmental relaxation. The two measures of segmental dynamics follow a similar trend. The dihedral autocorrelation probes larger segments and is slower at longer times.

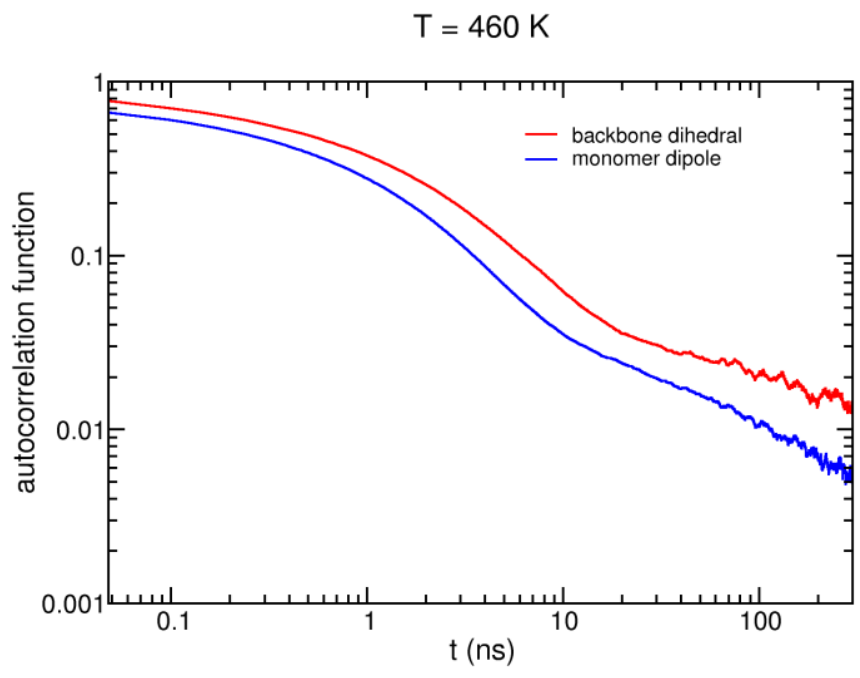

FIGURE S2 The monomer dipole and backbone dihedral autocorrelation functions for the thin film at $460 \mathrm{~K}$. 


\section{Comparison: Raw experimental and simulations data}

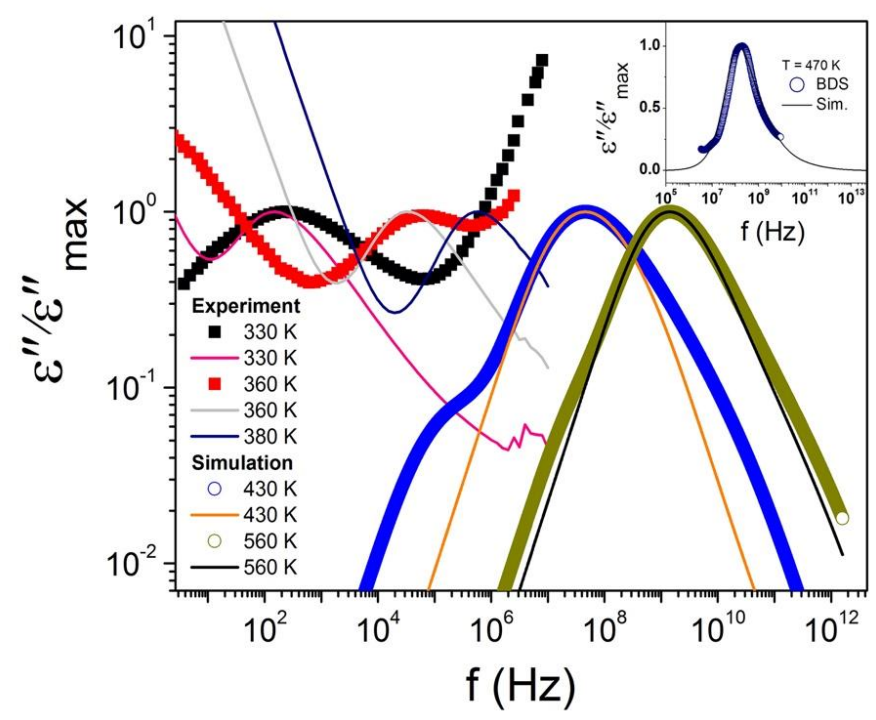

FIGURE S3. Dielectric loss as a function of frequency as determined experimentally and by simulation at different temperatures. Square and circle symbols represent data for thin films while the lines are bulk data. Inset: Simulation data for the bulk system compared to BDS data from Tyagi et al. ${ }^{1}$ at $470 \mathrm{~K}$.

\section{Analysis of dielectric data of the ultrathin polymer films}

The spectra for the thin films have more features arising from contributions of the thermally oxidized silica layer on the Si wafers, the silica nano-spacers, the polymeric film, the gap between the film layer and the spacers, and the wafers, with respective complex capacitances $C_{o}^{*}, C_{s p}^{*}, C_{f i l m}^{*}$ , $C_{g a p}^{*}$, and $C_{w}^{*}$. The total complex capacitance is therefore calculated by considering the serial arrangement of the constituent capacitors as

$$
\left(C_{\text {total }}^{*}\right)^{-1}=2\left(C_{w}^{*}\right)^{-1}+\left(C_{s p, e f f}^{*}\right)^{-1}+\left(C_{\text {gap }}^{*}\right)^{-1}+\left(C_{\text {film }}^{*}\right)^{-1}+\left(C_{o}^{*}\right)^{-1} \ldots \text { [Eq. S1] }
$$

where $C_{s p \text {,eff }}^{*}$ is the effective complex capacitance of the spacers and the free space between them, the two of them being in parallel arrangement to each other with respect to the applied field.

The total complex dielectric function that describes the measured data is

$$
\frac{D}{\varepsilon_{\text {total }}^{*}}=\frac{2 d_{w}}{\varepsilon_{w}^{*}}+\frac{d_{s p}}{\left(\rho_{s p} \varepsilon_{s p}^{*}+1-\rho_{s p}\right)}+\frac{d_{g a p}}{\varepsilon_{\text {gap }}^{*}}+\frac{d_{f i l m}}{\varepsilon_{\text {film }}^{*}}+\frac{d_{o}}{\varepsilon_{o}^{*}} \ldots[\text { Eq. S2] }
$$

where $D$ is the distance between the two electrodes, $\rho_{s p}$ is density of coverage of the nano-spacers, $d_{x}$ is a thickness with the respective subscripts having meanings already introduced. The thicknesses of the wafers, the nano-spacers, the oxide on the wafers and that of the thin films are known, in addition to setting $\varepsilon_{g a p}^{*}=1$, thus reducing the fit parameters. $\varepsilon_{\text {film }}^{*}$ is substituted with three Havriliak-Negami functions. Suffice it to mention that because it is experimentally not possible to determine the height of the gap, $d_{g a p}$, it is treated as a fit parameter in the analysis, which effectively means that we cannot accurately determine the relaxation strength of the polymeric layer. Several other modifications of Eq. S2 to account for more complicated experimental possibilities of this geometry have been considered - as comprehensively detailed in 
Tress et al. ${ }^{18}$ - and are found to deliver negligible changes to the shape parameters and position of the $\alpha$-peak obtained from the $\mathrm{HN}$ function of the polymeric layer.

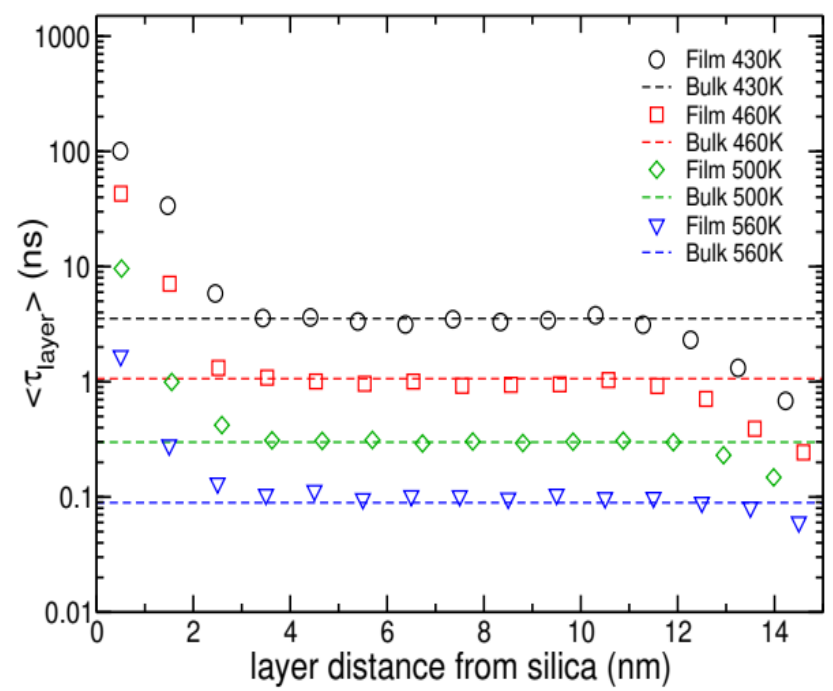

FIGURE S4. Average relaxation time for monomers at different film layers parallel to silica. The dashed lines represent the bulk relaxation time at each temperature.

We turn our focus now to the effect of the polymer/silica and the polymer/free vacuum interfaces on dynamics by systematically probing segmental motion in different layers throughout the film. The layers are defined based on the center of mass distance of the monomers from the silica surface and are each about $1 \mathrm{~nm}$ thick. The monomer dipole autocorrelation function $\left(\varphi_{\text {layer }}(\mathrm{t})\right)$ is calculated for monomers that are in the layer at time $\mathrm{t}$ from the total monomer dipole moment to improve the statistics. The average relaxation time for each layer $\left(<\tau_{\text {layer }}>\right)$ is calculated as $<$ $\tau_{\text {layer }}>=\int_{0}^{\infty} \varphi_{\text {layer }}(t) d t$ and presented in Figure S4 as a function of layer distance from the silica interface at different temperatures. Based on the profile of the relaxation times throughout the film, we define three main layers. Similar to the density profile (Fig. S8), the middle layer away from the two interfaces has bulk-like dynamics. The bottom layer (near the substrate) has much slower dynamics (more than 10 times slower than bulk); the thickness of this bottom layer is $\sim 4 \mathrm{~nm}$ and does not have a strong correlation with temperature. However, the contribution of the bottom layer to overall film dynamics increases with decreasing temperature since the fraction of monomers near the substrate increases with density. Finally, segmental relaxation is faster in the top layer (close to the vacuum interface), and its thickness and mole fraction increase at low temperatures. The relaxation time of the free segments near the vacuum approaches the bulk value at high temperatures hence the decrease in top layer thickness as defined here. Notably, the thickness of the layers as defined by dynamics is different from that determined from the density profile.

\section{Estimation of segmental dynamics from simulations}

Segmental relaxation can be estimated as a superposition of Debye relaxations. The autocorrelation function of bulk PVAc can be fitted with a Kohlrausch-Williams-Watts (KWW) function ${ }^{19}$ and the film can be fitted as a sum of two stretched exponentials that represent the separate processes: 


$$
\varphi_{K, \text { film }}=\alpha_{m} \exp \left(-\left(\frac{t}{\tau_{m}}\right)^{\beta_{m}}\right)+\alpha_{s} \exp \left(-\left(\frac{t}{\tau_{s}}\right)^{\beta_{s}}\right) \ldots \text { [Eq. S3] }
$$

where $\tau$ is the KWW relaxation time and $\beta$ describes the heterogeneity of the distribution of relaxation times. The $\alpha$ parameter in equation represents the weight of each term in the autocorrelation function. Using the KWW fits we extracted the average characteristic time $(<\tau\rangle)$ for each process:

$$
<\tau_{i}>=\frac{\alpha_{i}}{\beta_{\mathrm{i}}} \Gamma\left(\frac{1}{\beta_{\mathrm{i}}}\right) \tau_{i} \ldots \text { [Eq. S4] }
$$

where subscript $i$ represents the main bulk-like process $(m)$ or the slow process $(s)$.

The sum of $\alpha_{m}$ and $\alpha_{s}$ in Eq. S3 is less than 1 since we do not fit the initial relaxation caused by the fast-vibrational motions. The weight of the bulk-like relaxation, $\alpha_{m}$ is about 0.8 while the slow process has a small contribution $\left(\alpha_{s} \approx 0.15\right)$. The relaxation time of the first process $\left(\tau_{m}\right)$ has a similar value to bulk. As expected $\tau$ increases at lower temperatures for both processes but follows different temperature trends with $\tau_{s}$ approaching $\tau_{m}$ as the temperatures increase. The $\beta_{\mathrm{s}}$ parameter is generally lower than $\beta_{\mathrm{m}}$ as the dynamics become more heterogeneous close to the substrate. The bulk-like relaxation also become less heterogeneous at higher temperatures.

The complex dielectric permittivity, $\varepsilon^{*}$, in the frequency domain is the Fourier transform of the derivative of the dipole autocorrelation function

$$
\frac{\varepsilon^{*}-\varepsilon_{\infty}}{\Delta \varepsilon}=1-i \omega \int_{0}^{\infty} \frac{d \varphi(t)}{d t} \exp (-i \omega t) d t \ldots[\text { Eq. S5] }
$$

The calculated permittivity is normalized by the difference in permittivity at the infinite $\left(\varepsilon_{\infty}\right)$ and zero $\left(\varepsilon_{0}\right)$ limit of frequency. The equivalent loss $\left(\varepsilon^{\prime \prime}\right)$ profile is calculated as given in Eq. S6 using the distribution of relaxation times to directly compare the simulation results with BDS data.

$$
\frac{\varepsilon^{\prime \prime}}{\varepsilon_{\infty}-\varepsilon_{0}}=\sum_{\tau} \psi(\tau) \frac{\omega \tau}{1+(\omega \tau)^{2}} \ldots[\text { Eq. S6] }
$$

The measured and computed loss data for films is displayed at different temperatures in Figure S3. The simulation results show excellent agreement with BDS data for bulk at $470 \mathrm{~K}$ (see inset, Fig. $\mathrm{S} 3$ ). The simulation results in this figure underscore the fact that the slow relaxation, $\alpha_{\text {slow }}$, becomes more prominent with decreasing temperature. Also, while $\alpha_{\text {fast }}$ does not show up in the simulation data as a separate relaxation, there's evidence of broadening of the spectra on the high-frequency side, indicating the presence of new faster modes.

\section{Molecular weight dependence of segmental dynamics}

The segmental dynamics of PVAc oligomers depends on molecular weight until the density plateaus (at about 11000).$^{20}$ Figure S5 displays the total monomer dipole autocorrelation for the film and bulk at $560 \mathrm{~K}$ at two different chain lengths $\mathrm{N}=10$ monomers $(\mathrm{Mw}=860 \mathrm{~g} / \mathrm{mol}, \rho=0.945$ $\left.\mathrm{g} / \mathrm{cm}^{3}\right)$ and $\mathrm{N}=30\left(\mathrm{Mw}=2582 \mathrm{~g} / \mathrm{mol}, \rho=0.975 \mathrm{~g} / \mathrm{cm}^{3}\right)$. Both systems show a similar trend in dynamics with a bulk-like relaxation and a second slow at higher times. The dynamics in the $\mathrm{N}=30$ system is slower due to the higher density and the difference between two modes in film is more apparent. The average film relaxation time that is about 5 times larger than bulk for $\mathrm{N}=30$ and 1.6 time larger for $\mathrm{N}=10$. 


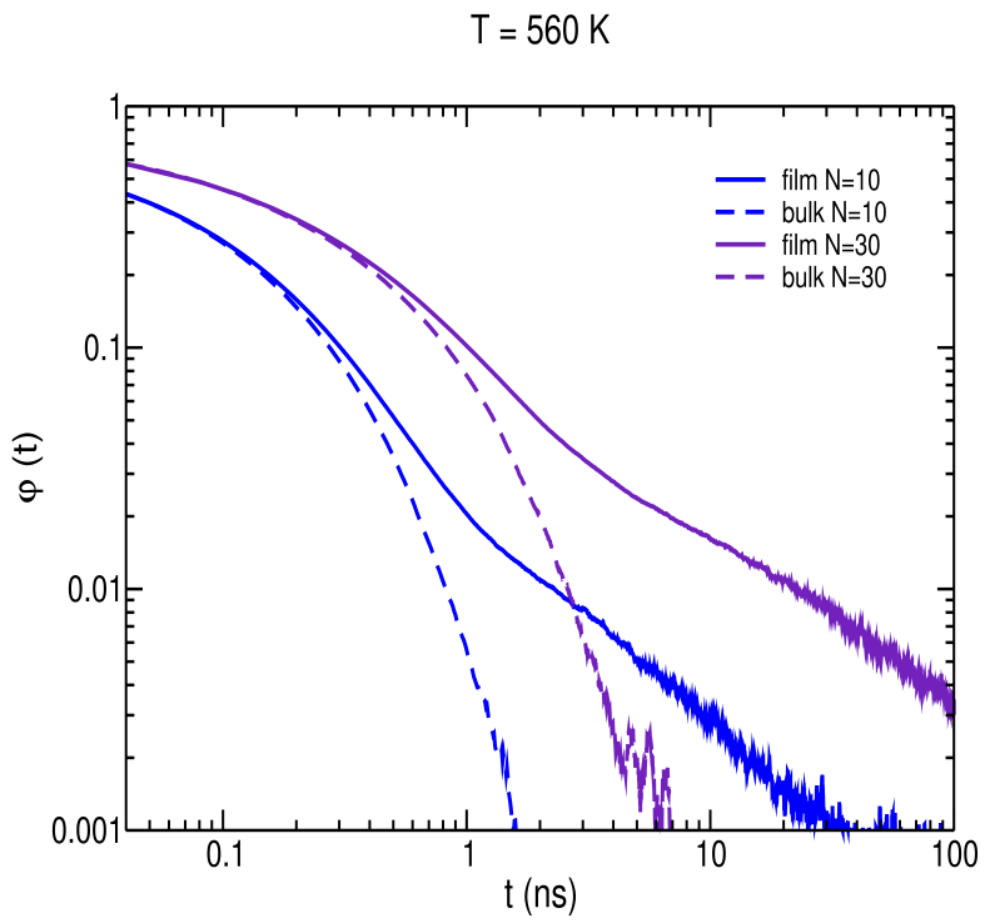

FIGURE S5. Segmental dynamics of film and bulk at two different chain lengths, $\mathrm{N}=10$ monomers and $\mathrm{N}=30$ monomers at $560 \mathrm{~K}$.

Figure S6 compares the single-chain dipole autocorrelation with monomer dipole autocorrelation for chains with $\mathrm{N}=10$ monomers at $560 \mathrm{~K}$. Probing the monomer dipole autocorrelation improves the statistics and enables a more detailed analysis of dynamics throughout the film thickness. The chain segmental relaxation is qualitatively similar to the monomer autocorrelation with an even slower decay for the adsorbed segments when cross-correlations between monomers are accounted for. The average relaxation time for chain dipole autocreation in film is 7.5 larger than bulk.

$$
\mathrm{T}=560 \mathrm{~K}
$$

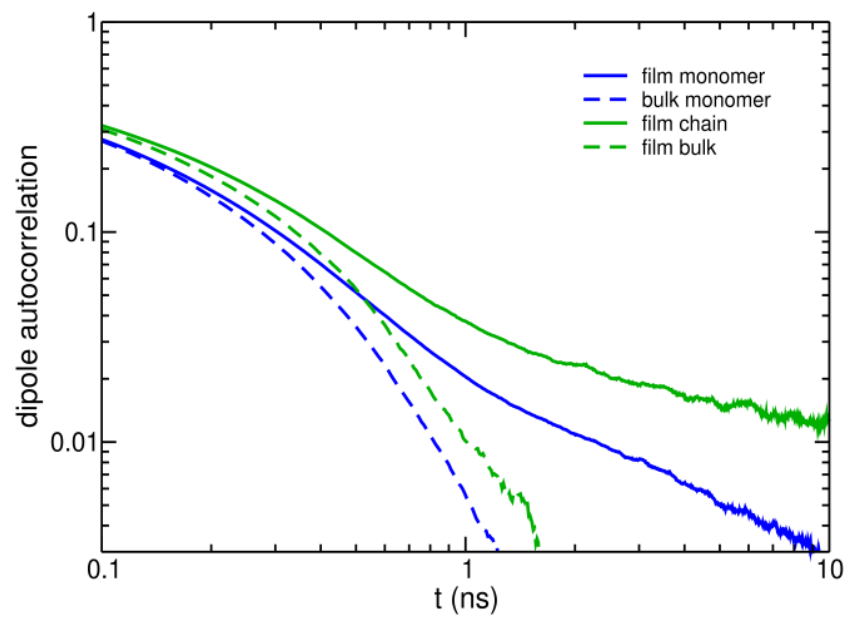

FIGURE S6. Monomer dipole autocorrelation and chain dipole autocorrelation for film and bulk for chains with $N=10$ monomers at 560 K. 


\section{Estimating the size of layers with modified mobility}

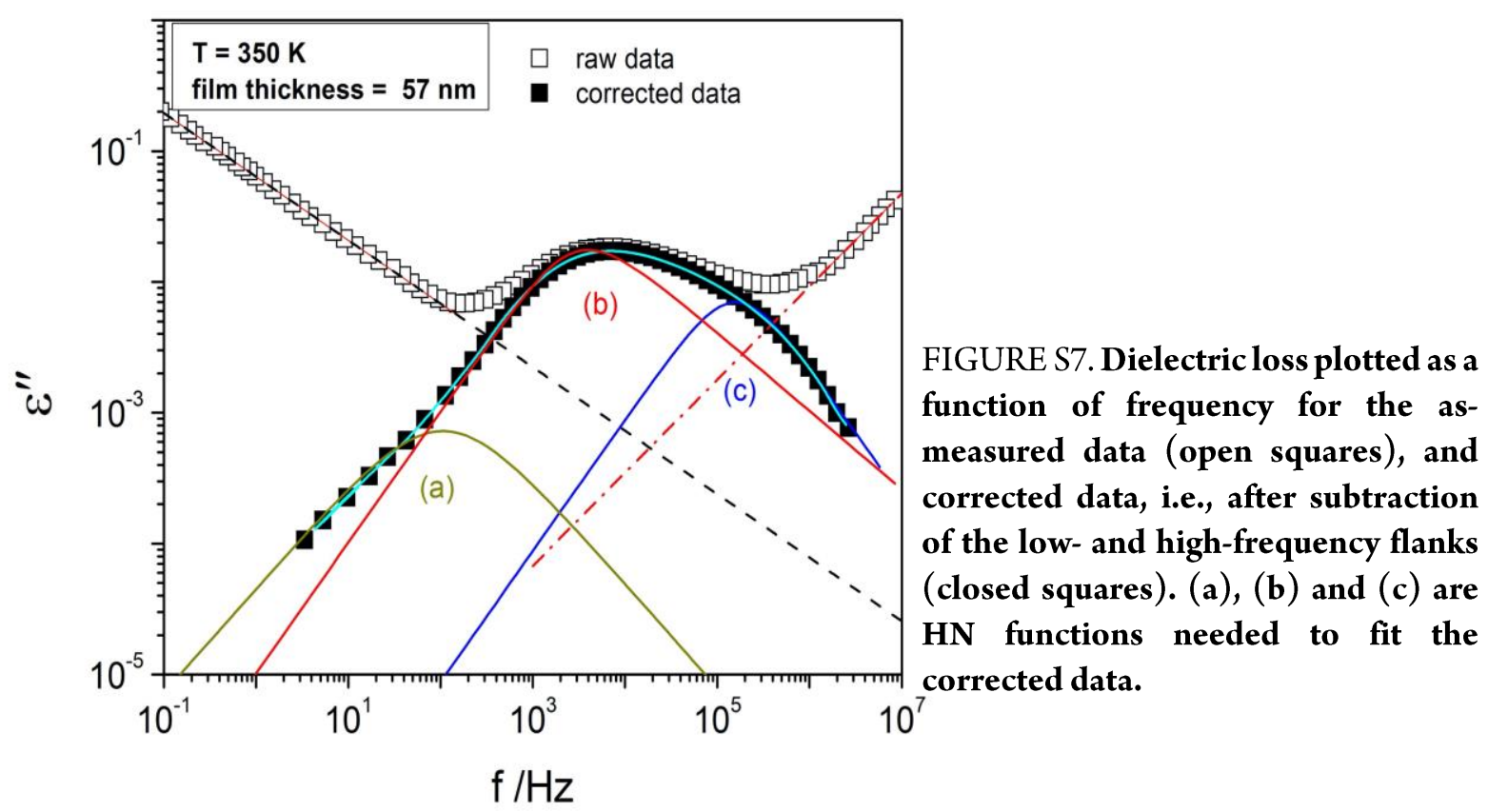

The conductivity contribution (low frequency flank) and that due to limited conductivity of the silicon electrodes (on the high-frequency side) were first subtracted from the measured dielectric loss. The former contribution is estimated by a linear function (dashed line) while the latter is described by a Debye-like function (dash-dotted line - which is the low-frequency wing of the said Debye-like function). Three Havriliak Negami (HN) functions are required to fit the corrected spectrum. These calculations were carried out on the data measured in a cooling run. Given that the overall film thickness is known from AFM measurements, the layers corresponding to processes (a), (b) and (c) in Figure S7 were assumed to be stacked together and estimated from the corresponding value of $\Delta \varepsilon$. For instance, for a film with overall thickness of $D \mathrm{~nm}$, the size of the layer, $d_{a}$, with slowed mobility is calculated as $d_{a}=D \Delta \varepsilon_{a} /\left(\Delta \varepsilon_{a}+\Delta \varepsilon_{b}+\Delta \varepsilon_{c}\right)$, where the subscripts derive from the representation in Figure S7. To estimate the uncertainty inherent in the obtained values of the layer sizes, this procedure was repeated for (some select) data measured in a heating run. The deviation from the arithmetic mean of the two values of layer thickness at a given temperature is taken as a representative measure of the error in our calculations. The error bars in Figure 5 (main paper) represent this random uncertainty.

In Figure S8, normalized dielectric strength for the three layers is plotted against inverse temperature. The values are normalized with respect to that found at the lowest accessed temperature $(330 \mathrm{~K})$. Since $\Delta \varepsilon$ is used to estimate layer thickness, the consistent trend seen in Figure S7 underscores the veracity of this approach. 


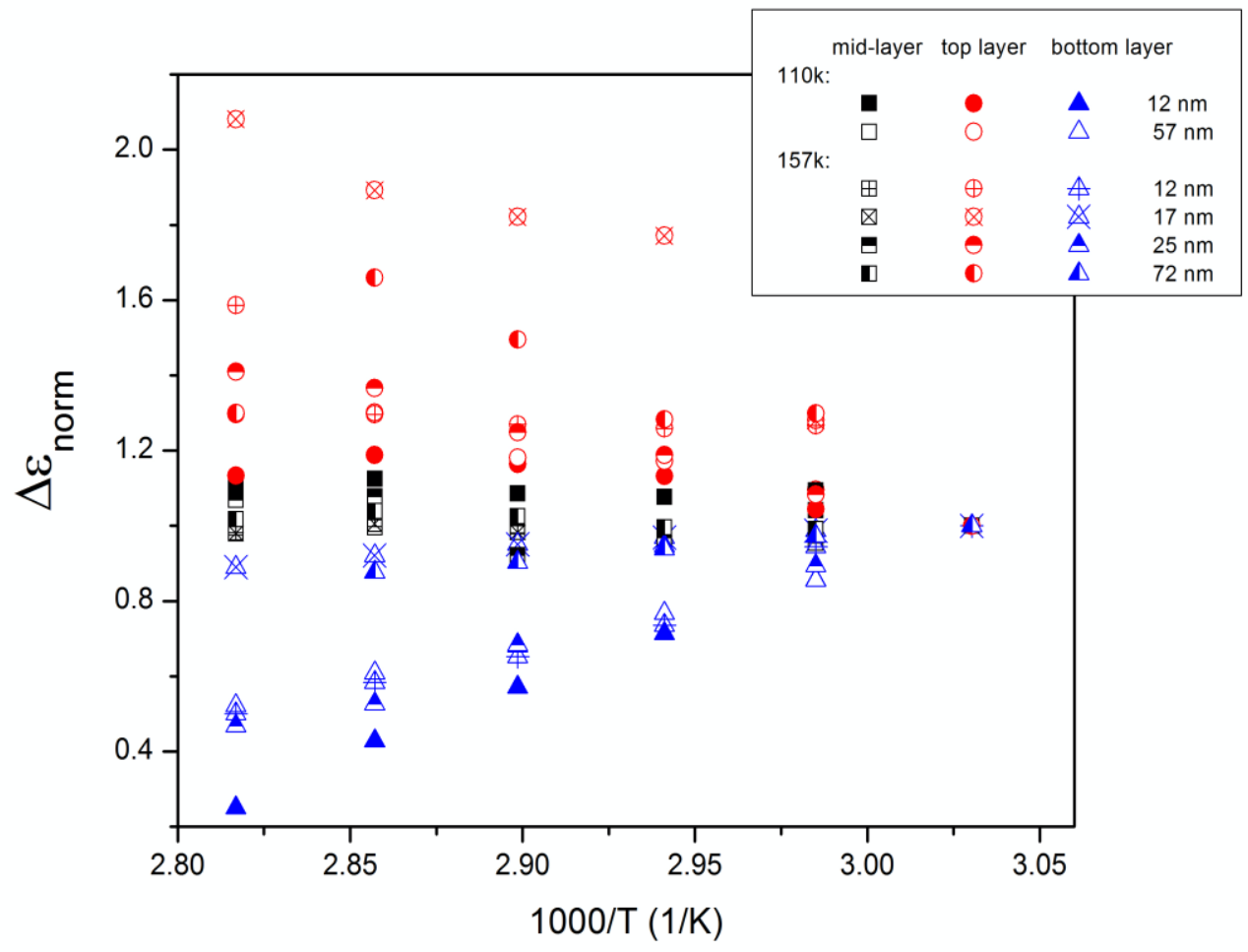

FIGURE S8. Dielectric relaxation strength for the three different layers (normalized with respect to the value at $330 \mathrm{~K}$ ) presented as a function of inverse temperature.

\section{Layer thickness from density (computation)}

Figure S9 shows the density profile for the PVAc film and bulk from MD simulations at 460 and $560 \mathrm{~K}$. The density is displayed as a function of distance from the substrate. Each bin displays the density for a layer normal to the $z$-direction with $\sim 0.1 \mathrm{~nm}$ thickness. Accumulation of segments near the attractive substrate results in a peak at the adsorbed layer ${ }^{21-25}$ at $\sim 0.7 \mathrm{~nm}$. The effect of the substrate on density is short-ranged and after $\sim 1.6 \mathrm{~nm}$ the density decays to a plateau that matches the bulk density. The drop in density from the bulk value at the top of the film marks the vacuum interface. 
[a]

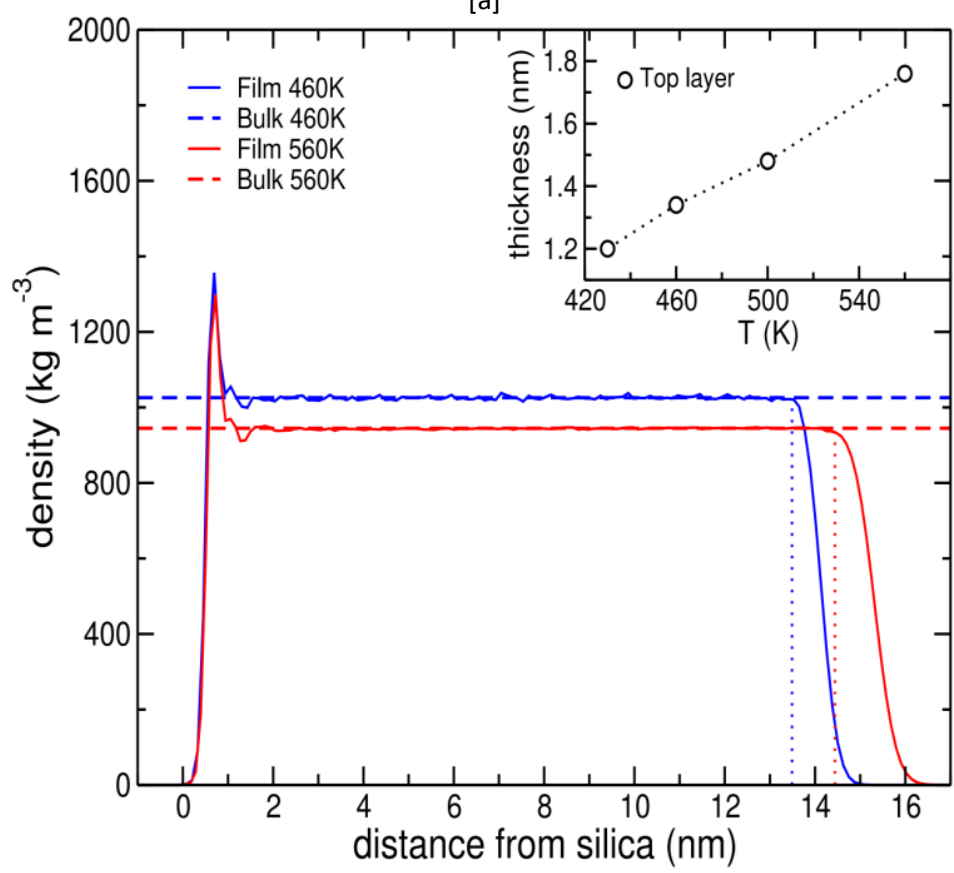

[b]

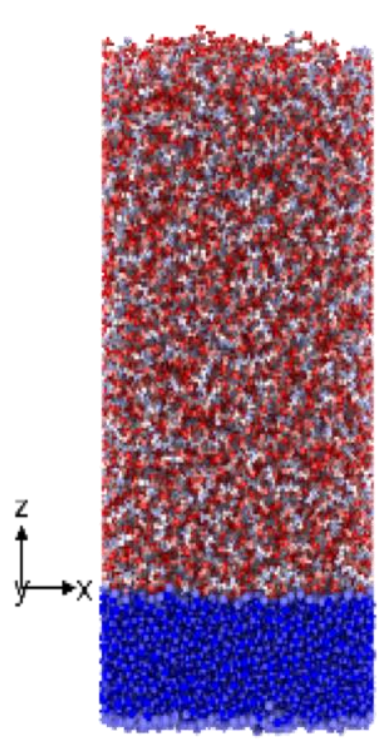

FIGURE S9. (a) Density profile of the film in the z-direction at $460 \mathrm{~K}$ (blue) and $560 \mathrm{~K}$ (red). The dashed lines represent the bulk density. The dotted lines separate the vacuum interface from hyperbolic tangent function fits. (b) shows a typical configuration of PVAc film on silica as employed in the simulation. Inset: estimate of the size of the top layer from density calculations.

The density decays to zero at the vacuum interface at the top of the film. This drop was fitted with the hyperbolic tangent function to determine the position of the interface (separated with dotted lines in Fig. S9).

$$
\rho(z)=\frac{\rho_{\text {bulk }}}{2}\left(1-\tanh \left(\frac{2\left(z-z_{e}\right)}{\delta}\right)\right) \ldots[\text { Eq. S7] }
$$

The width of the vacuum interface (equal to $2 \delta$ from the above equation) gradually expands with temperature as displayed in the inset of Fig. S9). The thickness of the absorbed layer as determined by the position of the first peak shows little change with temperature. 


\section{Layer thickness from dielectric relaxation strength (experimental)}

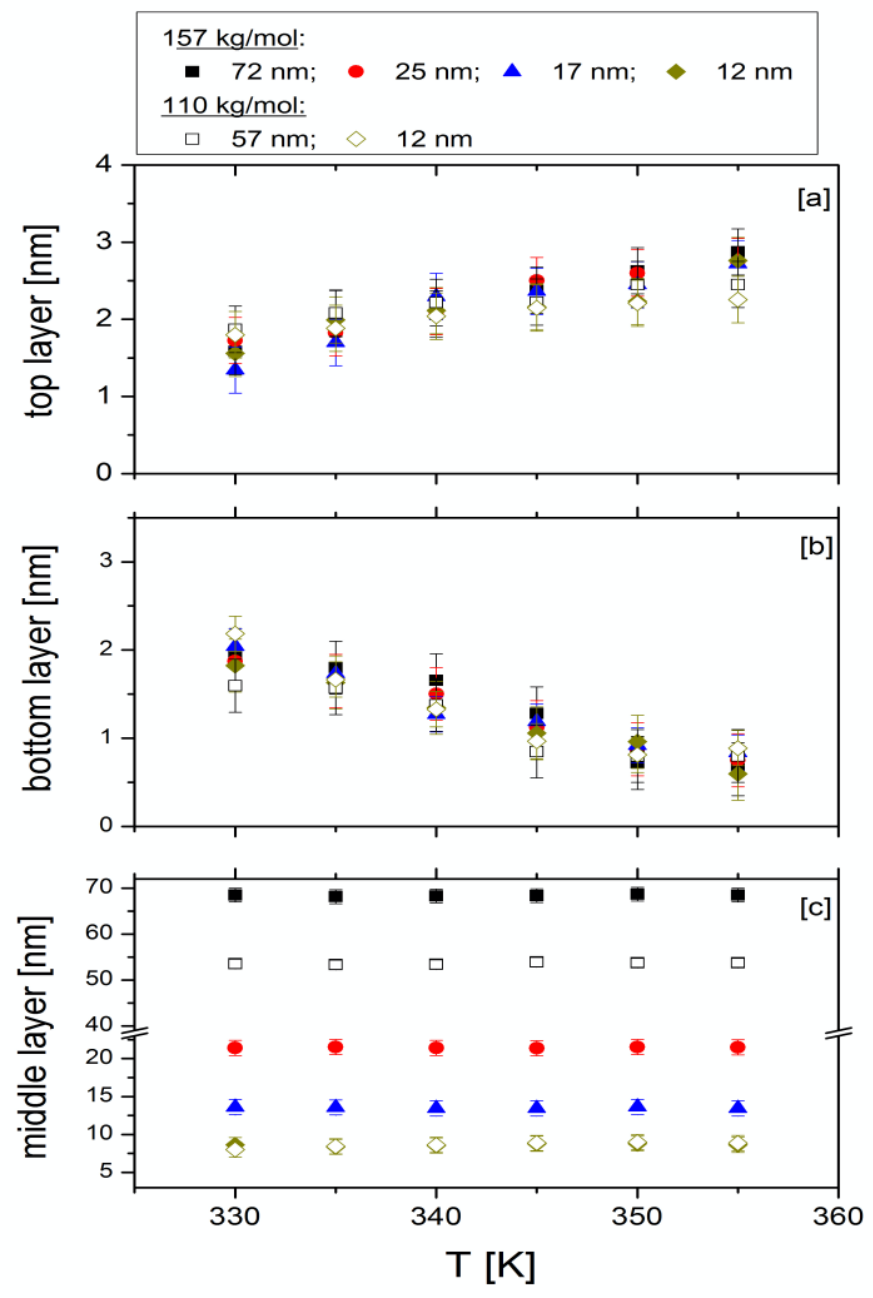

FIGURE S10. The size, in nanometers, of layers whose mobility is (a) increased (top layer), (b) slowed-downed (bottom layer) and (c) bulk-like (middle layer) expressed as functions of temperature for films prepared from two different molecular weights of PVAc, as indicated.

\section{References}

(1) Tyagi, M.; Alegria, A.; Colmenero, J. Heterogeneous Dynamics of Poly(vinyl acetate) Far Above Tg: A combined study by dielectric spectroscopy and quasielastic neutron scattering. J. Chem. Phys. 2005, 122 (24), 244909.

(2) Mapesa, E. U.; Tress, M.; Schulz, G.; Huth, H.; Schick, C.; Reiche, M.; Kremer, F. Segmental and chain dynamics in nanometric layers of poly(cis-1,4-isoprene) as studied by broadband dielectric spectroscopy and temperature-modulated calorimetry. Soft Matter 2013, 9 (44), 10592-10598.

(3) Blazevska-Gilev, J.; Spaseska, D. Thermal degradation of PVAc. J. Univ. Chem. Technol. and Metal. 2005, 40 (4), 287-290.

(4) Serghei, A.; Kremer, F. Broadband dielectric studies on the interfacial dynamics enabled by use of nanostructured electrodes. Rev. Sci. Instr. 2008, 79 (2), 026101.

(5) Tress, M.; Erber, M.; Mapesa, E. U.; Huth, H.; Müller, J.; Serghei, A.; Schick, C.; Eichhorn, K.-J.; Voit, B.; Kremer, F. Glassy dynamics and glass transition in nanometric thin layers of polystyrene. Macromolecules 2010, 43 (23), 9937-9944. 
(6) Mapesa, E. U.; Erber, M.; Tress, M.; Eichhorn, K. J.; Serghei, A.; Voit, B.; Kremer, F. Glassy dynamics in nanometer thin layers of polystyrene. Eur. Phys. J. Spec. Top. 2010, 189 (1), 173-180.

(7) Serghei, A.; Kremer, F. Broadband dielectric spectroscopy on ultrathin organic layers having one free (upper) interface. Rev. Sci. Instrum. 2006, 77 (11), 116108.

(8) Berendsen, H. J. C.; Spoel, D. v. d.; Drunen, R. v. GROMACS: A message-passing parallel molecular dynamics implementation. Comput. Phys. Commun. 1995, 91 (1), 43-56.

(9) Abraham, M. J.; Murtola, T.; Schulz, R.; Páll, S.; Smith, J. C.; Hess, B.; Lindahl, E. GROMACS: High performance molecular simulations through multi-level parallelism from laptops to supercomputers. SoftwareX 2015, 1-2, 19-25.

(10) Lopes, P. E. M.; Murashov, V.; Tazi, M.; Demchuk, E.; MacKerell, A. D. Development of an empirical force field for silica. Application to the quartz-water interface. J. Phys. Chem. B 2006, 110 (6), 2782-2792.

(11) Price, M. L. P.; Ostrovsky, D.; Jorgensen, W. L. Gas-phase and liquid-state properties of esters, nitriles, and nitro compounds with the OPLS-AA force field. J. Comput. Chem. 2001, 22 (13), 1340-1352.

(12) Ndoro, T. V. M.; Voyiatzis, E.; Ghanbari, A.; Theodorou, D. N.; Böhm, M. C.; Müller-Plathe, F. Interface of grafted and ungrafted silica nanoparticles with a polystyrene matrix: atomistic molecular dynamics simulations. Macromolecules 2011, 44 (7), 2316-2327.

(13) Pandey, Y. N.; Doxastakis, M. Detailed atomistic Monte Carlo simulations of a polymer melt on a solid surface and around a nanoparticle. J. Chem. Phys. 2012, 136 (9), 094901.

(14) Berendsen, H. J. C.; Postma, J. P. M.; van Gunsteren, W. F.; DiNola, A.; Haak, J. R. Molecular dynamics with coupling to an external bath. J. Chem. Phys. 1984, 81 (8), 3684-3690.

(15) Provencher, S. W. A Constrained regularization method for inverting data represented by linear algebraic or integral equations. Comput. Phys. Commun. 1982, 27, 213-227.

(16) Lide, D. R.; Milne, G. W. A. CRC Handbook of Data on Organic Compounds. CRC Press: 1993.

(17) Mark, J. E. Physical Properties of Polymers Handbook. Springer: Berlin, 2007; Vol. 1076.

(18) Tress, M.; Mapesa, E. U.; Kossack, W.; Kipnusu, W. K.; Reiche, M.; Kremer, F. Molecular dynamics in condensed (semi-) isolated polymer chains. In Advances in Dielectrics, Kremer, F., Ed. Springer: Heidelberg, 2014; pp 61-94.

(19) Williams, G.; Watts, D. C. Non-symmetrical dielectric relaxation behaviour arising from a simple empirical decay function. J. Chem. Soc. Faraday Trans. 1970, 66, 80-85.

(20) Negami, S.; Ruch, R. J.; Myers, R. R. The dielectric behavior of poly(vinyl acetate) as a function of molecular weight. J. Colloid Interface Sci. 1982, 90, 117-226.

(21) Rissanou, A. N.; Harmandaris, V. Dynamics of various polymer-graphene interfacial systems through atomistic molecular dynamics simulations. Soft Matter 2014, 10 (16), 2876-88.

(22) Mortazavian, H.; Fennell, C. J.; Blum, F. D. Structure of the interfacial region in adsorbed poly(vinyl acetate) on silica. Macromolecules 2015, 49 (1), 298-307.

(23) Mathieu, S.; Binder, K.; Paul, W. Dielectric relaxation of a polybutadiene melt at a crystalline graphite surface: atomistic molecular dynamics simulations. In Dynamics in Geometrical Confinement, Kremer, F., Ed. Springer International Publishing: Berlin, 2014; pp 1-15.

(24) Yelash, L.; Virnau, P.; Binder, K.; Paul, W. Slow process in confined polymer melts: layer exchange dynamics at a polymer solid interface. Phys. Rev. E Stat. Nonlin. Soft Matter Phys 2010, 82 (5 Pt 1), 050801.

(25) Yelash, L.; Virnau, P.; Binder, K.; Paul, W. Three-step decay of time correlations at polymer-solid interfaces. Europhys. Lett. 2012, 98 (2), 28006. 